\title{
Safety of Percutaneous Endoscopic Gastrostomy Tubes in Centenarian Patients
}

\author{
Zain A Sobani', Kevin Tin², Steven Guttmann ${ }^{3,4}$, Anna A Abbasi', Ira Mayer ${ }^{2,5}$ and Yuriy Tsirlin² \\ ${ }^{1}$ Department of Medicine, ${ }^{2}$ Division of Gastroenterology, Department of Medicine, Maimonides Medical Center, Brooklyn, NY, ${ }^{3}$ Long Island \\ Jewish Forest Hills, Queens, NY, ${ }^{4}$ Hofstra Northwell School of Medicine, Hempstead, NY, ${ }^{5}$ Albert Einstein College of Medicine, Yeshiva \\ University, Bronx, NY, USA
}

Background/Aims: Percutaneous endoscopic gastrostomy (PEG) is a relatively safe procedure; however, no study has evaluated the safety of PEG tube placement in patients over the age of 100 years.

Methods: We conducted a retrospective review of patient records for patients who underwent PEG tube placement. Thirty patients aged 100 years and older were identified and a random sample of 275 patients was selected for comparison.

Results: The mean age of the patients was $80.6 \pm 16.2$ years. No procedure-related deaths or major complications were identified; the overall inpatient mortality rate was $7.6 \%$. Minor complications were noted in $4 \%(n=12)$ of the patients. Centenarian patients were predominantly female ( $80 \%$ [ $n=24]$ vs. $54 \%$ [ $n=147], p=0.006)$, with a mean age of $100.5 \pm 0.9$ years. There was no significant difference in procedural success rates $(93.3 \%$ vs. $97.4 \%, p=0.222)$ or inpatient mortality $(6.7 \%[n=2]$ vs. $7.7 \%[n=21], p=1.000)$ between the two groups. However, a higher minor complication rate was noted in the older patients $(13.3 \%[n=4]$ vs. $2.9 \%[n=8], p=0.022)$.

Conclusions: Success rates, major complications and inpatient mortality associated with PEG tubes in patients aged over 100 years are comparable to those observed in relatively younger patients at our center; however minor complication rates are relatively higher. These findings lead us to believe that PEG tubes may be safely attempted in carefully selected patients in this subset of the population. Clin Endosc 2018;51:56-60

Key Words: Percutaneous endoscopic gastrostomy; Aged, 80 and over; Geriatric patients

\section{INTRODUCTION}

First described by Gauderer et al. in 1980, ${ }^{1}$ percutaneous endoscopic gastrostomy (PEG) is considered the gold standard for long-term enteral nutrition in patients unable to maintain adequate oral intake; with indications ranging from anatomical challenges such as head and neck malignancies to functional disorders resulting from neurological disease.

PEG is a relatively safe procedure, with a success rate of

Received: April 17, 2017 Revised: May 26, 2017

Accepted: June 11, 2017

Correspondence: Yuriy Tsirlin

Division of Gastroenterology, Department of Medicine, Maimonides Medical Center, 4802 10th Avenue, Brooklyn, NY 11219, USA

Tel: +1-718-283-6000, Fax: +1-718-283-6000, E-mail: ytsirlin@maimonidesmed.org

(cc) This is an Open Access article distributed under the terms of the Creative Commons Attribution Non-Commercial License (http://creativecommons.org/ licenses/by-nc/3.0) which permits unrestricted non-commercial use, distribution, and reproduction in any medium, provided the original work is properly cited.
95\%-98\%; ${ }^{2}$ however, it is associated with a risk of complications including but not limited to bleeding, aspiration, perforation of the aerodigestive tract, injury to surrounding structures, immediate or delayed site infections, and colocutaneous fistulae. ${ }^{2-4}$ A review of the literature shows a procedure-related mortality rate of $0.8 \%-1 \%$, with major complication rates ranging between $1 \%$ and $10 \%$, and minor complication rates ranging between $11 \%$ and $13 \%,{ }^{2,5-8}$ depending on the definition of major and minor complications.

Progressively older patients are undergoing PEG tube placements owing to an increasing life expectancy. Studies have shown that procedure-related mortality, in-hospital mortality, and one-month mortality are higher in older patients, ${ }^{9-11}$ especially in those aged 75 years and older. ${ }^{9}$ Although studies focusing on mortality have been reported, data regarding success rates and complications in the geriatric population is sparse. Further, to date, no study has evaluated the safety of PEG tube placement in patients 
aged 100 years or older. Therefore, we decided to conduct a retrospective audit of centenarian patients undergoing PEG tube placement at our facility, and evaluate the success rate, complications, and procedure-related mortality of PEG tube placement in this subset of the population.

\section{MATERIALS AND METHODS}

A retrospective review of patient records was carried out for patients aged 18 years and older who underwent PEG tube placement at our institution between July 1, 2011 and June 30, 2016. All patients who underwent PEG, regardless of the indication, were included in the study. The patients were divided into two subgroups based on their age at the time of the procedure. Group 1 included patients aged 100 years and above, whereas Group 2 included patients aged between 18 and 99 years at the time of the procedure. A total of 2,526 patients were identified, 30 of whom were aged 100 years and older. The 30 patients were included in Group 1; and the remaining 2,496 patients were included in Group 2. Assuming a minimal correlation of 0.20 , a total of 193 patients were required in Group 2 for achieving 80\% power, with an alpha of 0.05 . To maximize the power of the study, a random sample of 275 patients was selected from Group 2 using a computer-based random number generator, and the rest of the patients were excluded from the study.

All case notes, records, and investigations were reviewed, and the data were recorded in a predesigned database. Post-procedural complications were recorded along with the measures taken for their management. The complications were grouped into major (post-procedure aspiration, peritonitis, major bleeding requiring transfusion or intervention, colocutaneous fistulae, site infections, mucosal injuries requiring intervention and perforation) and minor (minor bleeding from the PEG site, mucosal injuries not requiring intervention, inadvertent tube removal within the index admission, tube leakage and blockage). Descriptive analysis was performed by calculating means and standard deviations for continuous variables, and proportions for categorical variables. Fischer's exact test was used to compare rates, and a $p$-value $<0.05$ was considered significant.

All PEG tube placements were performed by a credentialed attending gastroenterologist assisted by a fellow. The techniques were not standardized and were based upon the preferences of the attending gastroenterologist, and the individual cases. Sedation and airway were managed by our colleagues from the department of Anesthesiology or Pulmonary and Critical Care Medicine.

\section{RESULTS}

During the study period, a total of 2,526 patients underwent PEG tube placement at our institution, of which 30 were aged 100 years and older. We randomly selected $275 \mathrm{pa}$ tients from the remaining patients, using a random number generator for a comparison group. Three patients from the comparison group were excluded from the final analysis owing to lack of data, and a total of 302 patients were included in the study.

The mean age of the patients was $80.6 \pm 16.2$ years. Significant comorbidities are highlighted in Table 1 . The most common indication for PEG tube placement was dysphagia and aspiration, accounting for $58.3 \%(n=176)$ of the procedures. This was followed by cerebrovascular accidents $(n=53,17.5 \%)$, replacement of a malfunctioning PEG tube $(n=39,12.9 \%)$, and malnutrition with adult failure to thrive $(n=34,11.3 \%)$. Of the 176 patients with a primary indication of dysphagia and aspiration, 22 had an associated secondary indication of malnutrition and adult failure to thrive. Peri-procedural albumin was available for 244 patients, with a mean of $2.5 \pm 0.7 \mathrm{~g} / \mathrm{dL}$. Values measured within 30 days of the procedure were considered in the study.

In total, $89.1 \%(n=269)$ of the procedures were performed as inpatients, $9.9 \%(n=30)$ were performed as ambulatory procedures, and $1 \%(n=3)$ were planned as ambulatory procedures, but were later admitted for further monitoring. An overall procedural success rate of $97 \%$ was observed, with nine procedures aborted owing to lack of a suitable site or failure to intubate the esophagus. Procedure time was available for 176 patients; the median time from insertion of scope to withdrawal was 9 minutes. All times were rounded off to the next 30 seconds.

No major procedure-related complications were identified. Minor complications were noted in $4.0 \%(n=12)$ of the patients. The most common complications were minor mucosal injuries that did not require intervention (1.3\% $[n=4])$ and dislodged tubes $(1.3 \%[n=4])$, followed by minor bleeding $(0.7 \%[n=2])$, post-procedure fever $(0.7 \%[n=2])$ and superficial wound infection $(0.4 \%[n=1])$. There were no procedure-related deaths; however, the inpatient mortality was $7.6 \%(n=23)$ during the index hospitalization.

\section{Patients aged greater than 100 years}

The mean age of the patients in this subgroup was $100.5 \pm 0.9$ years. Women comprised a significantly larger proportion of this subgroup ( $80 \%$ [ $n=24]$ vs. $54 \%$ [ $n=147])$ in Group 2 ( $p=0.006)$. The indications were comparable to those in the overall data set, with dysphagia and aspiration accounting for $70 \%(n=21)$ of the procedures, followed by 
cerebrovascular accidents $(16.7 \%, n=5)$, malnutrition with adult failure to thrive $(6.7 \%, n=2)$, and replacement of malfunctioning PEG tube $(6.7 \%, n=2)$. The mean peri-procedureal albumin was $2.6 \pm 0.6 \mathrm{~g} / \mathrm{dL}$, compared to $2.5 \pm 0.7 \mathrm{~g} / \mathrm{dL}$ in the control group ( $p=0.738$ ). Data regarding the duration of the procedure was available for 18 patients. The median time from scope insertion to withdrawal was 9 minutes and 30 seconds. There was no significant difference in the procedural success rate $(93.3 \%$ vs. $97.4 \%, p=0.222)$ and inpatient mortality $(6.7 \%[n=2]$ vs. $7.7 \%[n=21], p=1)$ between

Table 1. Summary of Demographics, Comorbidities, Indications and Outcomes of the Patients in the Study

\begin{tabular}{|c|c|c|c|}
\hline & $\begin{array}{l}\text { Patients between 18-99 yr } \\
\qquad(n=275)\end{array}$ & $\begin{array}{l}\text { Patients aged } 100+y r \\
\qquad(n=30)\end{array}$ & $p$-value \\
\hline \multicolumn{4}{|l|}{ Demographics } \\
\hline Mean age & $78.4 \pm 15.6$ & $100.5 \pm 0.9$ & \\
\hline Female & $54 \%(147)$ & $80.0 \%(24)$ & 0.006 \\
\hline Inpatient & $89.7 \%(244)$ & $83.3 \%(25)$ & \\
\hline Outpatient & $9.6 \%(26)$ & $13.3 \%(4)$ & \\
\hline Outpatient converted to inpatient & $0.7 \%(2)$ & $3.3 \%(1)$ & \\
\hline Albumin (mg/dL) & $2.5 \pm 0.7$ & $2.6 \pm 0.6$ & 0.738 \\
\hline \multicolumn{4}{|l|}{ Indications } \\
\hline Dysphagia and aspiration & $50 \%(136)$ & $60.0 \%(18)$ & \\
\hline Dysphagia/Aspiration/Faliure to thrive & $7.0 \%(19)$ & $10.0 \%(3)$ & \\
\hline Cerebrovascular accident & $17.6 \%(48)$ & $16.7 \%(5)$ & \\
\hline Failure to thrive & $11.8 \%(32)$ & $6.7 \%(2)$ & \\
\hline Replacement & $13.6 \%(37)$ & $6.7 \%(2)$ & \\
\hline \multicolumn{4}{|l|}{ Comorbidities } \\
\hline Diabetes mellitus & $29.4 \%(80)$ & $13.3 \%(4)$ & \\
\hline Hypertension & $64.3 \%(175)$ & $86.7 \%(26)$ & \\
\hline Hyperlipidemia & $29.4 \%(80)$ & $33.3 \%(10)$ & \\
\hline Coronary artery disease & $27.9 \%(76)$ & $40.0 \%(12)$ & \\
\hline Congestive heart failure & $18.0 \%(49)$ & $36.7 \%(11)$ & \\
\hline Chronic kidney disease & $8.5 \%(23)$ & $3.3 \%(1)$ & \\
\hline Chronic obstructive pulmonary disease & $9.9 \%(27)$ & $10 \%(3)$ & \\
\hline Atrial fibrillation & $22.1 \%(60)$ & $13.3 \%(4)$ & \\
\hline Aortic stenosis & $2.6 \%(7)$ & $10 \%(3)$ & \\
\hline Hypothyroidism & $14.3 \%(39)$ & $13.3 \%(4)$ & \\
\hline \multicolumn{4}{|l|}{ Complications } \\
\hline Mucosal injury & $0.4 \%(1)$ & $6.7 \%(2)$ & \\
\hline Minor bleeding & $0.4 \%(1)$ & $3.3 \%(1)$ & \\
\hline Dislodged tube & $1.1 \%(3)$ & $3.3 \%(1)$ & \\
\hline Superficial wound infection & $0.4 \%(1)$ & - & \\
\hline Fever & $0.7 \%(2)$ & - & \\
\hline Any complication & $2.9 \%(8)$ & $13.3 \%(4)$ & 0.022 \\
\hline Death (unrelated) & $7.7 \%(21)$ & $6.7 \%(2)$ & 1.000 \\
\hline Unsuccessful procedures & $2.6 \%(7)$ & $6.7 \%(2)$ & \\
\hline Success rate & $97.4 \%$ & $93.3 \%$ & 0.222 \\
\hline Procedure time $(\mathrm{min})^{\mathrm{a})}$ & $9.0 \pm 3.5$ & $9.5 \pm 13.0$ & \\
\hline
\end{tabular}

${ }^{a)}$ Procedure time was available for 176 patients; 18 in the group aged 100 years and older and 158 in the group aged between 18 and 99 years. 


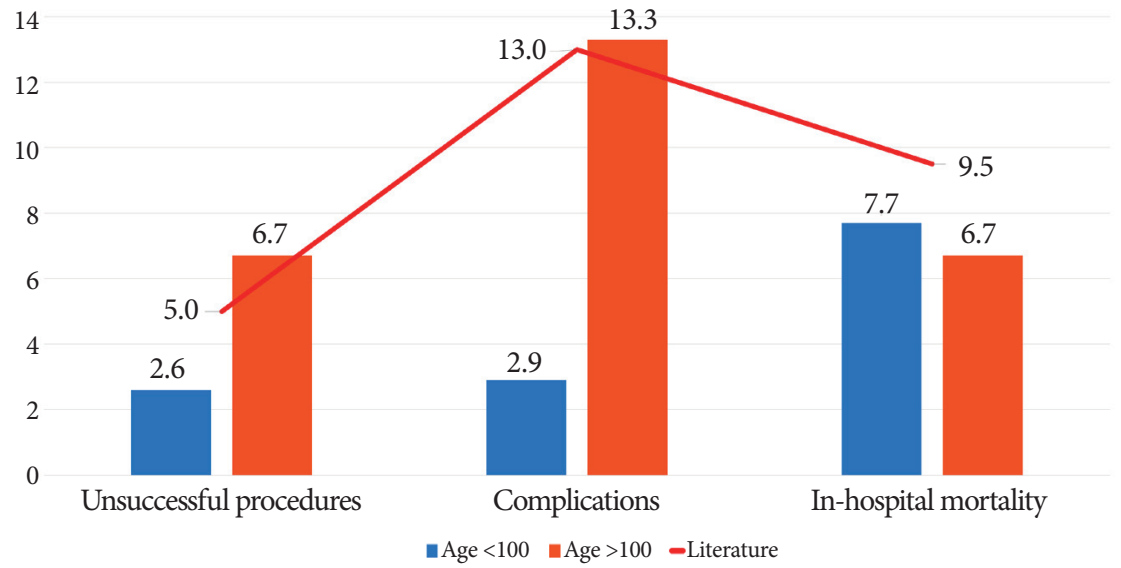

Fig. 1. Rates of unsuccessful procedure $(p=0.222)$, complications $(p=0.022)$, and in-hospital mortality $(p=1)$ between the two groups, as well as those reported in the literature. Note that the data from literature is reported as ranges in the discussion; the upper limit of the range is plotted on the graph. Statistical analysis is only between our patients, data from literature is plotted for illustrative purposes. patients aged over 100 years, and those aged between 18 and 99 years. However, a higher rate of minor complications was noted in the older patients $(13.3 \%[n=4]$ vs. $2.9 \%[n=8]$, $p=0.022$ ) (Fig. 1). Two patients had minor mucosal injuries that did not require intervention, one developed minor bleeding at the site that resolved without intervention, and one had a tube that was inadvertently dislodged.

\section{DISCUSSION}

Geriatric patients are prone to develop dysphagia requiring PEG tube placement, owing to neurodegenerative disorders, cerebrovascular accidents, and malignancies. ${ }^{12}$ Studies have shown higher procedure-related, in-hospital, and onemonth mortality in older patients, ${ }^{9-11}$ especially in those aged 75 years and older. ${ }^{9}$

Owing to increases in life expectancy, the number of centenarian patients is increasing; however, no specific data regarding this subset of the population are available. In our retrospective review, we identified 30 patients aged over 100 years who underwent PEG tube placement at our facility between July 1, 2011 and June 30, 2016. Although there is no validated tool for assessing life expectancy in patients aged 100 years and older, as a policy, the procedure was offered only to patients who were deemed to have a life expectancy of at least 30 days via clinical assessment of the patients by the primary clinical team. The patients were predominantly female, which could likely be attributed to the increased life expectancy of women in the US. ${ }^{13}$ Despite having significant comorbidities, the patients tolerated the procedure well. Our procedural success rate of $93.3 \%$ was comparable to that of the controls (97.4\%) and to the $95 \%-98 \%$ success rate reported in literature for younger patients. ${ }^{2,5,14,15}$ Of the two patients in whom PEG tube placement was unsuccessful, esophageal intubation could not be achieved in the first patient, where- as the second patient had a large hiatal hernia and a safe puncture site could not be identified as a result of the hernia. Techniques using spinal needles or $5 \mathrm{Fr}$ drainage needles in conjunction with fluoroscopic guidance, ${ }^{3,16}$ and direct visualization and reduction of the hernia have been described in patients with hiatal hernias requiring PEG. ${ }^{17}$ However, our team did not feel comfortable attempting these techniques in these patients. The first patient subsequently underwent interventional radiology-guided gastrostomy tube placement and the second patient underwent a laparoscopic gastrostomy tube placement.

No major procedure-related complications were observed in either of the groups. The centenarian group had a higher minor complication rate of $13.3 \%$ compared to the corresponding rate of $2.9 \%$ observed in the control group; however, this was comparable to the minor complication rate of $11 \%-13 \%$ reported in the general population. ${ }^{2,5-8}$ In the centenarian group, two patients had minor mucosal injuries, one patient had minor bleeding from the site that resolved without intervention, and one patient had an inadvertently dislodged tube. Of the two patients who had mucosal injuries, one patient was undergoing replacement of a malfunctioning PEG tube. The patient had an esophageal stricture and required the use of an overtube to remove the old bumper. The second patient had an uneventful procedure, but was noted to have had a small mucosal tear at the esophagogastric junction at the end of the procedure, which was likely the result of endoscopic manipulation. None of the patients required any intervention, and both patients had an uneventful post-procedural course. The limited number of complications (total 12, with 8 in the control group) made it difficult to reliably evaluate the potential factors that may have predisposed the patients to minor complications. Given the paucity of patients aged 100 years and above who undergo PEG tube placement, it may not be possible to obtain an adequate sample to estimate such a relationship. 
The overall inpatient mortality was $7.6 \%(n=23)$. There was no significant difference in the mortality between the two groups: the centenarian group had an inpatient mortality of $6.7 \%(n=2)$, compared to $7.7 \%(n=21)$ in the control group. Both groups had lower inpatient mortality rates than the $9.0 \%-9.5 \%$ reported in literature. ${ }^{11,18}$ Of the two patients in the centenarian group who died within the index hospitalization, the first was being managed for a large thalamic hemorrhage resulting in hemiplegia, while the second was being managed for severe sepsis secondary to urinary tract infection and concomitant cholecystitis. Both patients had uneventful procedures, but died from progression of their underlying disease. Both patients had advance directives for "do not resuscitate" and "do not intubate" prior to their deaths. These cases reiterate the need for careful patient selection and timing for PEG tube placements.

It is important to consider that by virtue of the location of our hospital and the communities that we serve, our patients tend to be older. The mean age of the patients in the control or "younger" group was $78.4 \pm 15.6$ years. However, our success, complication, and inpatient mortality rates were comparable to those reported in literature.

Although the sample size in our study was small, given the limited number of patients aged above 100 years who require PEG tube placement, our success and inpatient mortality rates are comparable to those of relatively younger patients at our center and to those reported in literature. Although minor complication rates, although higher than the rates observed in the control group, they are comparable to rates reported in literature for younger patients. Thus, we can speculate that PEG tube placement may be safely attempted in carefully selected patients in this subset of the population; however, larger studies may be required to further validate our findings.

\section{Conflicts of Interest}

The authors have no financial conflicts of interest.

\section{REFERENCES}

1. Gauderer MW, Ponsky JL, Izant RJ Jr. Gastrostomy without laparotomy: a percutaneous endoscopic technique. J Pediatr Surg 1980;15:872-875.
2. McClave SA, Chang WK. Complications of enteral access. Gastrointest Endosc 2003;58:739-751.

3. Chang WK, Hsieh TY. Safety of percutaneous endoscopic gastrostomy in high-risk patients. J Gastroenterol Hepatol 2013;28 Suppl 4:118-122.

4. DeLegge MH, Saltzman JR, Lipman TO, Robson KM. Gastrostomy tubes: complications and their management [database on the Internet]. Alphen aan den Rijn: Wolters Kluwer Health, UpToDate; 2015 [updated 2015 Apr 15; cited 2017 Mar]. Available from: https://www.uptodate. $\mathrm{com} /$ contents/gastrostomy-tubes-complications-and-their-management?source=search_result\&search $=\% 22$ Gastrostomy $\% 20$ tubes: $\% 20$ complications\%20and\%20their\%20management $\% 22 \&$ selectedTitle $=1 \sim 1$.

5. Larson DE, Burton DD, Schroeder KW, DiMagno EP. Percutaneous endoscopic gastrostomy. Indications, success, complications, and mortality in 314 consecutive patients. Gastroenterology 1987;93:48-52.

6. Keung EZ, Liu X, Nuzhad A, Rabinowits G, Patel V. In-hospital and long-term outcomes after percutaneous endoscopic gastrostomy in patients with malignancy. J Am Coll Surg 2012;215:777-786.

7. Rimon E. The safety and feasibility of percutaneous endoscopic gastrostomy placement by a single physician. Endoscopy 2001;33:241-244.

8. Wollman B, D’Agostino HB, Walus-Wigle JR, Easter DW, Beale A. Radiologic, endoscopic, and surgical gastrostomy: an institutional evaluation and meta-analysis of the literature. Radiology 1995;197:699-704.

9. Arora G, Rockey D, Gupta S. High in-hospital mortality after percutaneous endoscopic gastrostomy: results of a nationwide population-based study. Clin Gastroenterol Hepatol 2013;11:1437-1444.e3.

10. Light VL, Slezak FA, Porter JA, Gerson LW, McCord G. Predictive factors for early mortality after percutaneous endoscopic gastrostomy. Gastrointest Endosc 1995;42:330-335.

11. Wirth R, Voss C, Smoliner C, Sieber CC, Bauer JM, Volkert D. Complications and mortality after percutaneous endoscopic gastrostomy in geriatrics: a prospective multicenter observational trial. J Am Med Dir Assoc 2012;13:228-233.

12. Callahan CM, Haag KM, Weinberger M, et al. Outcomes of percutaneous endoscopic gastrostomy among older adults in a community setting. J Am Geriatr Soc 2000;48:1048-1054.

13. Gorman BK, Read JG. Why men die younger than women. Geriatr Aging 2007;10:182-191.

14. Pender SM, Courtney MG, Rajan E, McAdam B, Fielding JF. Percutaneous endoscopic gastrostomy--results of an Irish single unit series. Ir J Med Sci 1993;162:452-455.

15. Wilson WR, Hariri SM. Experience with percutaneous endoscopic gastrostomy on an otolaryngology service. Ear Nose Throat J 1995;74:760762.

16. Manjunath RS, Fisher NC. Percutaneous endoscopic gastrostomy tube placement in patients with compound hiatus hernia and intrathoracic stomach: a case series. Gut 2011;60 Suppl 1:A98-A99.

17. Xenos ES. Percutaneous endoscopic gastrostomy in a patient with a large hiatal hernia using laparoscopy. JSLS 2000;4:231-233.

18. Gumaste VV, Bhamidimarri KR, Bansal R, Sidhu L, Baum J, Walfish A. Factors predicting early discharge and mortality in post-percutaneous endoscopic gastrostomy patients. Ann Gastroenterol 2014;27:42-47. 